\title{
APLICAÇÃO DA TÉCNICA DELPHI NA CONSTRUÇÃO DE INDICADORES DE SUSTENTABILIDADE
}

Silvano da Silva Coutinho ${ }^{2}$

Angela Maria Magosso Takayanagui ${ }^{3}$

RESUMO: Os indicadores de sustentabilidade são ferramentas importantes em diferentes contextos da sociedade, podendo auxiliar os gestores públicos no planejamento da gestão, na execução de políticas públicas e na tomada de decisões. A construção de indicadores de sustentabilidade impulsionam discussões sobre um novo modelo de desenvolvimento que visa garantir as necessidades do presente sem comprometer o futuro, e as ações fundamentadas nas informações levantadas a partir da aplicação desses indicadores permitem a análise do desempenho ao longo do tempo e possibilitam previsões futuras. Este trabalho apresenta reflexões de como a técnica Delphi pode ser aplicada para a construção de indicadores de sustentabilidade. A técnica Delphi pode ser realizada em diversas situações, nesse estudo discutiremos sua aplicação em três etapas, discursando sobre as dificuldades e os benefícios que podem ser encontrados a partir de sua utilização para a construção de indicadores. A proposta final dos indicadores é obtida a partir do consenso de especialistas da área do estudo proposto, devendo-se deixar claro a quem são destinados, a fim de buscar uma aproximação da aplicação

\footnotetext{
${ }^{1}$ Doutoranda em Ciências, Programa de Pós-graduação em Enfermagem em Saúde Pública, Universidade de São Paulo - USP. Engenheira Civil. tati.veiga@yahoo.com.br.

${ }^{2}$ Doutor em Ciências, Programa de Pós-graduação em Enfermagem em Saúde Pública, Universidade de São Paulo - USP. Docente da Universidade do Centro-Oeste do Paraná _ UNICENTRO.

silvano.unicentro@gmail.com.

${ }^{3}$ Doutora em Enfermagem. Universidade de São Paulo - USP. Professora Associada da Escola de Enfermagem de Ribeirão Preto (EERP/USP). amtakay@eerp.usp.br.
} 
da teoria com a atuação prática dos profissionais. Para analisar o consenso entre os especialistas, pode ser utilizada a análise descritiva em cada uma das etapas da técnica proposta, resultando na elaboração de um painel com o resumo dos dados obtidos. A partir dessa discussão foi possível perceber características importantes que diferenciam a técnica Delphi de outras técnicas tradicionais para a construção de indicadores.

Palavras-chave: Indicadores. Sustentabilidade. Técnica Delphi.

\section{INTRODUÇÃO}

Os indicadores desempenham muitas funções e são úteis para comunicar idéias, pensamentos e valores. Eles podem levar a melhores decisões e ações mais eficazes por simplificar, clarear e apresentar as informações de forma agregada para os tomadores de decisão política, a fim de medir e calibrar o progresso em direção às metas de desenvolvimento sustentável que visa garantir as necessidades do presente sem comprometer o futuro (CNUMAD, 2001).

Oferecem, também, um aviso prévio para evitar retrocessos econômicos, sociais e ambientais (UNITED NATIONS, 2007). Dessa forma, os indicadores são componentes essenciais na avaliação dos progressos, no sentido desse modelo de desenvolvimento (GALLOPÍN, 1997).

O debate referente a esse novo conceito de desenvolvimento não se restringe apenas à esfera do ambiente, mas abrange áreas da economia, ética e planejamento urbano, entre outras (TAKAYANAGUI, 1993). Segundo Araújo e Carvalho (2011), "a sustentabilidade assumiu, no final do século XX, um papel chave na reflexão em torno do modelo de desenvolvimento econômico vigente e seus impactos sociais e ambientais tanto em escala regional quanto global".

Os indicadores de sustentabilidade são importantes ferramentas de mensuração e avaliação do progresso rumo ao desenvolvimento sustentável, sendo necessários para subsidiar a elaboração de políticas públicas e a tomadas de decisões (BELLEN, 2006; MEADOWS, 1998). 
A Comissão para o Desenvolvimento Sustentável (CSD) lidera um movimento internacional com a participação de governos, instituições acadêmicas, organizações não governamentais (ONGs), organizações do sistema das Nações Unidas e especialistas do mundo todo voltadas para a construção de indicadores. Esse movimento revela a importância dos indicadores e busca por em prática os capítulos 8 e 40 da Agenda 21, referente à necessidade de informações para a tomada de decisões (CNUMAD, 2001; VEIGA, 2010).

De acordo com Polaz e Teixeira (2009), um dos desafios na busca por esse tipo de desenvolvimento consiste no estabelecimento de técnicas capazes de prover informações para facilitar a avaliação do grau de sustentabilidade das sociedades. Nesse sentido, o processo de elaboração dos indicadores deve ser participativo, envolvendo especialistas na área em diferentes setores da sociedade, considerando o fato de que cada pessoa traz um conjunto de habilidades distintas, possibilitando uma definição conjunta de objetivos e metas para a elaboração dos indicadores (HODGE; HARDI; BELL, 1999; PHILIPPI JR; MALHEIROS; AGUIAR 2005).

Partindo dessas reflexões, o presente trabalho tem como objetivo discutir a aplicação da técnica Delphi para a construção de indicadores de sustentabilidade que pode ser aplicado em diferentes áreas do conhecimento.

\section{TÉCNICA DELPHI}

O termo Delfos tem origem na mitologia grega, residia no tempo de Apolo com "poder de transferir desejos dos deuses e visões do futuro aos mortais inquietos (GONTIJO, 2007), e as primeiras publicações que mencionam o uso dessa técnica datam de 1948. A versão moderna da técnica Delphi foi desenvolvida baseando-se na intuição de grupos para ampliar projeções individuais, sendo utilizada por peritos da Rand Corporation durante o período de guerra fria para analisar e prever os acontecimentos na Força Aérea (SPÍNOLA, 1984).

Em diferentes estudos podem ser encontradas terminologias diferentes para explicar a metodologia utilizada. Os autores referem-se tanto ao método Delphi quanto à 
técnica Delphi e, em relação aos estágios, podem ser chamados de rounds, turnos, etapas, entre outros. Neste estudo, será utilizada a denominação: técnica Delphi, sendo indicadas suas diferentes etapas.

A estratégia metodológica fundamenta-se em um processo de comunicação que permite a um grupo de especialistas versarem sobre problemas complexos, situando-se na interação de três elementos: o grupo coordenador, os participantes e o(s) instrumento(s) (COUTINHO, 2011).

A técnica Delphi consiste em uma técnica de processo grupal que tem por finalidade obter, comparar e direcionar o julgamento de especialistas para um consenso sobre a área de estudo, promovendo a convergência de opiniões (SPÍNOLA, 1984), sendo destacada sua efetividade ao permitir que um grupo de indivíduos, como um todo, possa lidar com um problema complexo. Dentre as suas características, ressalta-se o respeito e a valorização da experiência e do conhecimento dos sujeitos participantes, que, de uma forma dirigida, são colocados a uma apreciação do grupo de sujeitos.

Segundo Linstone e Turoff (2002), a aplicação dessa técnica já foi utilizada no setor público e privado, destacando-se em vários campos, como indústria, planejamento social, educação e saúde, entre outros (DESLANDES et al., 2010; WITT, 2005; RIBEIRO; HELLER, 2011; PADILHA, 2009).

Vários autores têm destacado a importância e as vantagens da utilização da técnica Delphi em pesquisas científicas. Segundo Wright e Giovinazzo (2000), pelo fato da técnica poder ser realizada por correio ou meio eletrônico, possibilita maior adesão dos especialistas que participam de acordo com suas disponibilidades de tempo, reduzindo consideravelmente o custo comparado à realização de participação presencial.

Os procedimentos utilizados na técnica Delphi incluem a seleção dos especialistas e permite a consulta de um amplo grupo de sujeitos através da aplicação de questionários, a fim de se obter uma convergência das respostas dos sujeitos participantes, na intenção de alcançar um consenso que possa representar a consolidação do julgamento do grupo (GONTIJO, 2007).

Essa técnica permite ainda realizar previsões em situações de carência de dados que possam balizar as ações necessárias para se adequar à política nos diversos setores, de modo a fazer, por exemplo, com que uma lei "saia do papel" e se torne algo "vivo" (WRIGHT; GIOVINAZZO, 2000). 
Essa técnica também é eficaz para o tratamento de questões complexas, uma vez que permite ao especialista contribuir com informações ou julgamentos sobre uma área do problema de âmbito muito maior do que o campo de sua especialidade (LISTONE; TUROFF, 2002), pautando-se em opinião especializada para auxiliar na tomada de decisões sobre práticas, necessidades e metas (THOMAS; NELSON; SILVERMAN, 2007).

O estudo Delphi é flexível na sua concepção, isto permite a coleta de dados mais rica que conduz a uma compreensão mais profunda de questões complexas e requerem o conhecimento de pessoas que entendem as diferentes questões econômicas, sociais e políticas (OKOLI; PAWLOWSKI, 2004), sendo particularmente valorizada pela sua capacidade de estruturar e organizar a comunicação do grupo (POWELL, 2002).

Outro fator importante refere-se à garantia do anonimato dos sujeitos que eliminam a influência de fatores como "status" acadêmico ou profissional, possibilitando uma participação mais ativa e menos "enviesada" (WRIGHT; GIOVINAZZO, 2000), estimulando a expressão de opiniões sinceras, não influenciadas por pressão do grupo ou outros fatores extrínsecos. Dessa forma, ela provê consenso de opinião dos experts sem o viés que pode ocorrer em técnicas grupais em que os membros podem ser intimidados, sendo também reduzidos fatores restritivos, como a supressão de posições minoritárias em grupos (WITT, 2005).

Kaio e Securato (1997) reforçam a importância do anonimato ao ressaltar que essa característica pode auxiliar na superação de alguns fatores sociológicos que afetam a interação grupal numa reunião presencial, como a diminuição da persuasão por parte de pessoas com maior capacidade de eloquência; cada respondente tem a chance de reavaliar seu ponto de vista e possibilita que pensamentos heterodoxos e/ou audaciosos sejam expressos.

Assim, a possibilidade de representação estatística da distribuição dos resultados e o "feedback" das respostas para reavaliação nas rodadas subsequentes representa uma das suas principais características, que permite aos sujeitos redefinir seus julgamentos baseado na visão coletiva do grupo (LINSTONE; TUROFF, 2002). A aplicação de sucessivas etapas da técnica permite que pontos de vista possam ser retirados, alterados ou adicionados a partir da reflexão dos especialistas. 
Para Okoli e Pawlowski (2004), a técnica Delphi caracteriza-se como uma forte metodologia e apresenta um quadro comparativo que contrasta os pontos da aplicação da técnica Delphi versus a abordagem tradicional em pesquisas acadêmicas (Figura 1).

\begin{tabular}{|ll} 
Critérios de avaliação & Pesquisa tradicional \\
\hline Resumo de & Os pesquisadores projetam um \\
questionário com questões relevantes \\
para a questão do estudo. Engloba \\
inúmeras questões sobre a validade das \\
perguntas que eles devem considerar \\
para desenvolver uma boa pesquisa. O \\
questionário pode incluir perguntas que \\
solicitam dados quantitativos ou \\
qualitativos, ou ambos. Os \\
investigadores decidem na população \\
sobre quais as hipóteses levantadas, e \\
seleciona uma amostra aleatória dessa \\
população para administrar o \\
levantamento. Os entrevistados \\
preenchem e devolvem o questionário. \\
Os pesquisadores, então, analisam as \\
respostas para investigar as questões da \\
pesquisa.
\end{tabular}

Representatividade da Amostra

Tamanho da amostra para poder estatístico e resultados significativos

Individual versus resposta do grupo estatística, os pesquisadores selecionam, aleatoriamente, uma amostra que é representativa da população de interesse.

Uma vez que o objetivo é a generalização de resultados para uma maior população, os pesquisadores precisam selecionar uma amostra de tamanho que seja suficientemente grande para detectar estatisticamente efeitos significativos na população. Análise de poder é necessária para determinar um tamanho de amostra.

Os pesquisadores calculam a média para as respostas individuais para determinar a média das resposta por amostra, a qual generalizam para a população do estudo.
Usando técnicas de amostragem

\section{Estudo Delphi}

Todas as questões projetadas em um questionário também se aplicam no estudo Delphi. Depois que os pesquisadores elaboram o questionário, eles selecionam um grupo apropriado de reconhecidos especialistas para responder as perguntas. $\mathrm{O}$ pesquisador, então, administra o levantamento e análise das respostas. Em seguida, o pesquisador projeta outro levantamento, baseado nas primeiras respostas, pedindo aos respondentes para reverem suas respostas originais e/ou responder a outras perguntas com base no feedback do grupo. Os pesquisadores reiteram esse processo até alcançarem um grau satisfatório de consenso. Os entrevistados são mantidos em anonimato durante todo o processo.

As perguntas que um estudo Delphi investiga são de elevada incerteza e especulação. Assim, uma população geral, ou mesmo um subconjunto restrito de população, não pode ser suficientemente experiente para responder perguntas com precisão. Um estudo Delphi corresponde a um painel virtual de especialistas para chegar a resposta a uma pergunta difícil. Assim, um estudo Delphi pode ser considerado um tipo de reunião virtual ou uma decisão de grupo técnico.

O tamanho do grupo Delphi não depende do poder da estatística, mas sim na dinâmica de grupo para se chegar a um consenso entre os especialistas.

Para questões que requerem parecer de peritos, a média de respostas individuais é inferior a média gerada por processos de decisão em grupo; senso essa uma das características da técnica Delphi. 
Confiabilidade e Revisão de Resposta
Um critério importante para pesquisas de avaliação é a confiabilidade das medidas. Pesquisadores realizam o préteste e o reteste, para assegurar testereteste.
O pré-teste é também uma garantia de confiabilidade importante para o método Delphi. No entanto, o teste-reteste não é relevante, uma vez que os pesquisadores têm oportunidade de reavaliar suas respostas.

\section{Figura 1 - Quadro comparativo de pesquisa tradicional com a técnica Delphi}

Fonte: Adaptado de Okoli e Pawlowski (2004).

\begin{tabular}{|c|c|c|}
\hline Critérios de avaliação & Pesquisa tradicional & Estudo Delphi \\
\hline Validade do Construto & $\begin{array}{l}\text { Em um método tradicional, segundo os } \\
\text { autores, a validade de construção é } \\
\text { assegurada pelo projeto cuidadoso da } \\
\text { pesquisa e pela realização do pré-teste. }\end{array}$ & $\begin{array}{l}\text { Além do que é exigido em uma } \\
\text { pesquisa, o método Delphi pode } \\
\text { empregar uma validação adicional pela } \\
\text { construção, pedindo aos especialistas } \\
\text { para validar a interpretação do } \\
\text { pesquisador e categorizar as variáveis. } \\
\text { Pelo fato da técnica Delphi não ser } \\
\text { anônimo (o pesquisador) permite esta } \\
\text { etapa de validação, ao contrário de } \\
\text { muitas pesquisas. }\end{array}$ \\
\hline Anonimato & $\begin{array}{l}\text { Os entrevistados são quase sempre } \\
\text { anônimos uns para com os outros, e, } \\
\text { muitas vezes anônimo para o } \\
\text { pesquisador. }\end{array}$ & $\begin{array}{l}\text { Respondentes são sempre anónimos } \\
\text { uns aos outros, mas nunca anônimos } \\
\text { para o pesquisador. Isto dá aos } \\
\text { pesquisadores mais oportunidade de } \\
\text { acompanhar e esclarecer as dúvidas. }\end{array}$ \\
\hline $\begin{array}{l}\text { "Não-resposta" de } \\
\text { questões }\end{array}$ & $\begin{array}{l}\text { Os pesquisadores precisam investigar a } \\
\text { possibilidade de viés de não-resposta } \\
\text { para garantir que a amostra continua a } \\
\text { ser representativa da população. }\end{array}$ & $\begin{array}{l}\text { Não-resposta é normalmente muito } \\
\text { baixa em inquéritos Delphi, pois a } \\
\text { maioria dos pesquisadores obteve a } \\
\text { respostas dos sujeitos quanto a sua } \\
\text { participação. }\end{array}$ \\
\hline "Efeitos de Atritos" & $\begin{array}{l}\text { Para levantamentos individuais, atrito } \\
\text { (abandono de participante) não é um } \\
\text { problema. Para estudos "multipasso" de } \\
\text { levantamentos repetidos, pesquisadores } \\
\text { devem investigar o desgaste para } \\
\text { assegurar que é aleatória e não } \\
\text { sistemática. }\end{array}$ & $\begin{array}{l}\text { Semelhante ao de não-resposta, o atrito } \\
\text { tende a ser baixo em estudos Delphi, e } \\
\text { os pesquisadores geralmente podem } \\
\text { determinar a causa pelo fato de falarem } \\
\text { com os sujeitos sobre as desistências. }\end{array}$ \\
\hline Riqueza de dados & $\begin{array}{l}\text { A riqueza dos dados depende da forma e } \\
\text { profundidade das perguntas, e da } \\
\text { possibilidade de acompanhamento } \\
\text { (entrevistas, questionários). O } \\
\text { acompanhamento é, muitas vezes, } \\
\text { limitado quando não há disponibilidade } \\
\text { dos pesquisadores. }\end{array}$ & $\begin{array}{l}\text { Além das questões de riqueza das } \\
\text { pesquisas tradicionais, estudos Delphi } \\
\text { inerentemente proporcionam dados } \\
\text { mais ricos devido as suas várias } \\
\text { iterações e à sua revisão de resposta } \\
\text { devido ao feedback. }\end{array}$ \\
\hline
\end{tabular}

Figura 1 - Quadro comparativo de pesquisa tradicional com a técnica Delphi (continuação) Fonte: Adaptado de Okoli e Pawlowski (2004). 


\subsection{Etapas da técnica Delphi}

A técnica Delphi pode ser aplicada em diferentes etapas ou estágios. Neste estudo discutiremos a sua aplicação em três etapas.

\section{Primeira etapa}

Nessa etapa, serão selecionados pesquisadores/profissionais da área de estudo para atuarem como especialistas na pesquisa. A fim de facilitar a participação dos sujeitos pode ser elaborada uma lista preliminar de indicadores da área de estudo fundamentada em referências atualizadas.

É importante destacar que, em algumas situações essas referências podem ser escassas e, nesse caso, poderia acrescentar uma etapa onde os sujeitos informariam os indicadores, para ser realizada uma análise qualitativa antes da primeira etapa proposta neste estudo. Destaca-se que esse procedimento pode dificultar a adesão dos especialistas, considerando que os sujeitos devem ser selecionados por serem "experts" na área do estudo e, normalmente, são pessoas com muitos compromissos profissionais.

Após realizar o convite, aos sujeitos que aceitarem participar da pesquisa deve ser encaminhado um primeiro instrumento para a coleta de dados com a lista preliminar de indicadores. Para verificar a relevância desses indicadores, pode ser aplicada a classificação por meio da escala "Likert" na qual o participante escolhe uma entre várias respostas escalonadas, possibilitando maior amplitude de expressão dos especialistas para avaliar o nível de concordância ou discordância em relação às afirmações a eles apresentadas, representando o grau de aceitação dos indicadores.

A escala proposta para a realização dessa etapa foi baseada no estudo de Linstone e Turoff (2002). Esta classificação foi utilizada para avaliar a relevância de indicadores em diferentes áreas, com a mensuração do grau de importância definida em cinco níveis (PADILHA, 2009; RIBEIRO; HELLER, 2011):

$>$ 5. Muito importante;

> 4. Importante;

> 3. Desejável;

> 2. Não prioritário; 
>1. Dispensável

Na primeira etapa da aplicação da técnica Delphi, poderá ser sugerida, no final do instrumento, uma nova redação para o texto apresentado para os indicadores propostos, assim como a inclusão de novos indicadores que os participantes considerem importantes e acreditem não terem sido contemplados no instrumento de coleta de dados apresentado.

Deve ser estabelecido um prazo para devolução do questionário. Após esse período estabelece-se o contato com os sujeitos que não tiverem devolvido 0 questionário.

A partir do recebimento de todos os questionários, os dados são organizados em planilhas, realizando-se a análise estatística descritiva, a fim de avaliar o grau de consenso entre os especialistas para os indicadores da lista preliminar. Os indicadores considerados com elevado nível de consenso serão aqueles que atingirem o terceiro quartil na análise, ou seja, correspondem àqueles que forem indicados por, pelo menos, $75 \%$ dos sujeitos com a designação de nível 4 ou 5 (PADILHA, 2009; RIBEIRO; HELLER, 2011).

Os indicadores adicionados ou alterados pelos sujeitos devem ter seu texto tratado qualitativamente, de modo a categorizar as respostas segundo a similaridade ou equivalência dos dados, ou análise de conteúdo.

A partir da análise de dados será elaborado um novo instrumento, incluindo os indicadores informados pelos especialistas.

\section{Segunda etapa:}

A partir da análise dos dados obtidos na primeira etapa, deve ser encaminhado um novo instrumento aos especialistas na segunda etapa da técnica Delphi, juntamente com a tabulação dos resultados obtidos na etapa anterior. Dessa forma, será possibilitado um "feedback" a todos os participantes, onde os sujeitos terão a oportunidade de interagir com a visão de outros especialistas que, embora com diferentes formações e oriundos de diversas localizações geográficas, tem em comum uma determinada área de estudo, possibilitando a reavaliação do grau de importância atribuído aos indicadores, somando a essa construção, uma imprescindível visão multidisciplinar. 
Uma grande vantagem dessa técnica consiste que o envio e o retorno do instrumento podem ser realizados via e-mail ou correio, não sendo necessário o contato presencial como em muitas técnicas, com realização de contato telefônico somente com os participantes que não cumprirem o prazo de retorno.

A partir do retorno dos dados da segunda etapa, será novamente realizada a análise dos dados para estabelecimento de consenso entre as respostas obtidas, utilizando-se os mesmos critérios apresentados na primeira etapa.

\section{Terceira etapa:}

Haverá, então, o terceiro e último envio do instrumento aos participantes. Nessa etapa, pode ser disponibilizado um espaço ao final do instrumento onde o participante deverá descrever sua justificativa para uma designação de nível 3 ou inferior para qualquer um dos indicadores. Nesse momento, espera-se que os sujeitos possam destacar fatores relevantes que não tenham sido abordados pelos demais especialistas durante o processo. Ao final do retorno dos questionários, os dados serão organizados e tabulados, por meio da estatística descritiva.

A partir dos dados obtidos nas três etapas da aplicação da técnica, deve ser elaborado um painel que apresentará um panorama geral dos resultados obtidos em todas as etapas, com a apresentação uma lista de indicadores que obtiveram o consenso entre os especialistas da área, com diferentes formações e provenientes de várias regiões geográficas, promovendo um caráter multidisciplinar na construção dos indicadores.

O fluxograma (Figura 2) apresenta um resumo da aplicação da técnica Delphi em três etapas para elaboração de indicadores. 
Elaboração do instrumento com a lista preliminar de indicadores de sustentabilidade

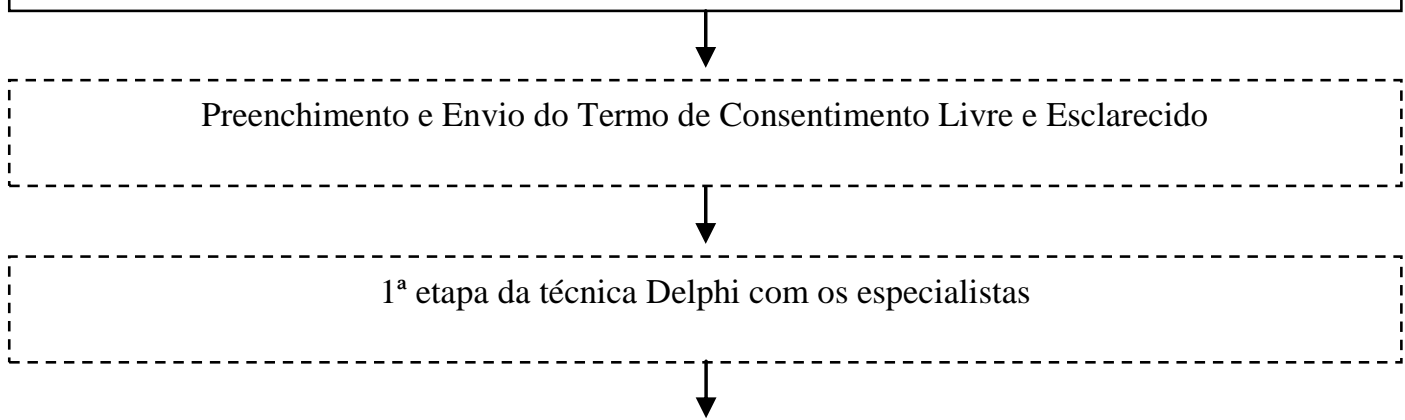

Tabulação dos dados dos instrumentos recebidos após a $1^{\mathrm{a}}$ etapa

Categorização dos novos indicadores segundo a similaridade dos dados ou análise de conteúdo Análise dos dados obtidos na $1^{\mathrm{a}}$ etapa

Elaboração do segundo questionário com a listagem dos indicadores de sustentabilidade e a Escala Likert

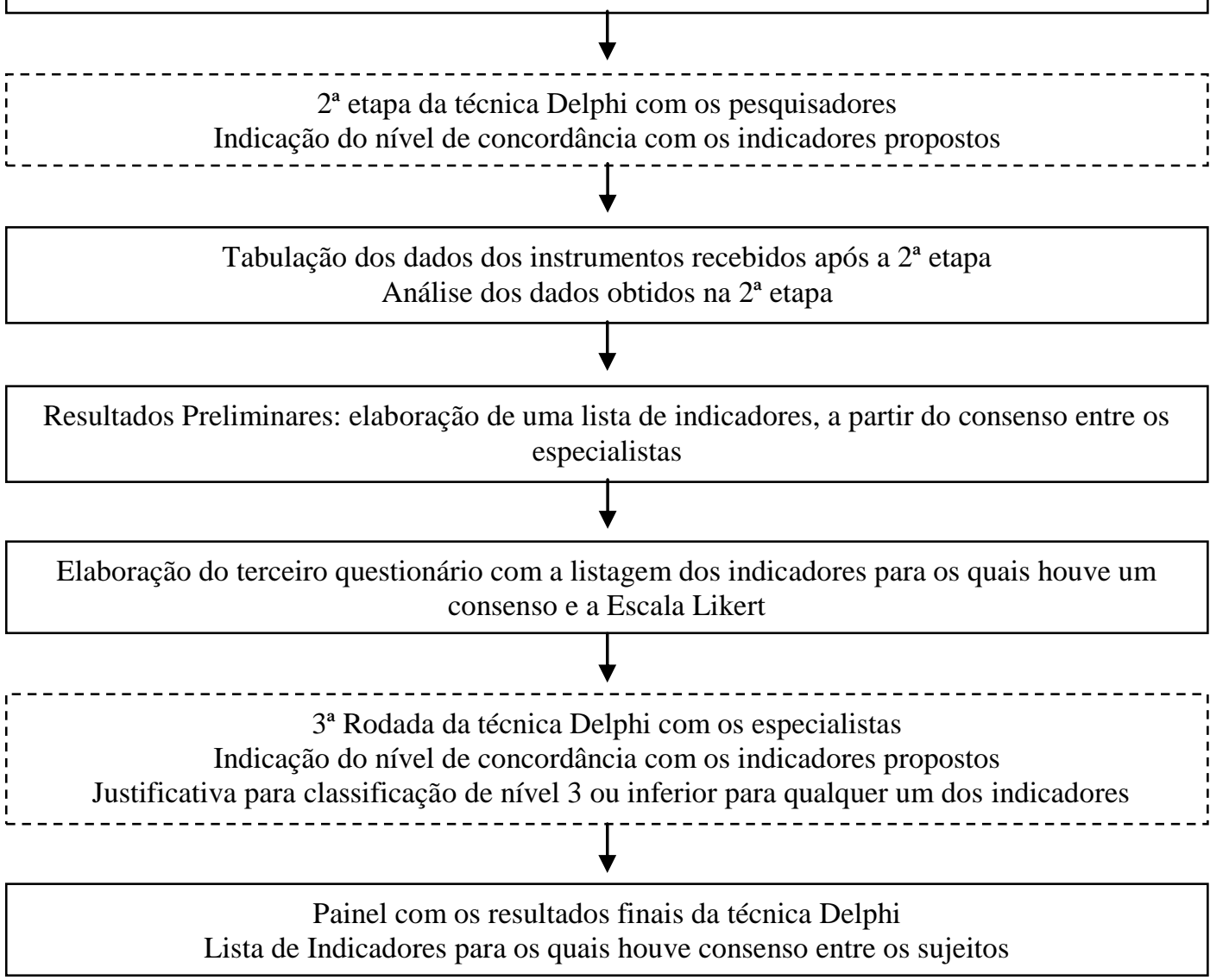

Figura 2 - Fluxograma de execução da técnica Delphi

Fonte: Adaptado de Wright; Giovinazzo (2000). 
A elaboração do painel Delphi auxilia na análise dos resultados obtidos nas diferentes etapas da pesquisa que deve ser realizada em conjunto, visando observar o desenvolvimento do estudo de uma forma global, ao mesmo tempo em que não são desconsideradas as particularidades específicas de cada etapa, a fim de dar subsídio para uma análise e discussão consistente dos dados.

\section{CONSIDERAÇÕES FINAIS}

A técnica Delphi é uma ferramenta de pesquisa versátil e possibilita aos pesquisadores a realização de uma investigação em diferentes etapas segundo os objetivos que se deseja alcançar no estudo.

Muitas vantagens podem ser destacas na aplicação dessa técnica, conforme apresentado e discutido neste trabalho. Destaca que uma das principais vantagens consiste na possibilidade de realizar previsões em situações em que há carência de dados históricos.

Outra vantagem que pode ser destacada corresponde a uma ampla participação de especialistas na área do estudo, localizados em diferentes áreas regionais, sem a necessidade de sua presença física, fator esse que minimiza o índice de recusa e possibilita a realização da investigação com um menor investimento de recursos financeiros.

O anonimato também corresponde a uma vantagem importante que possibilita a participação de especialistas sem a a influência de fatores como "status" acadêmico ou profissional, destacando-se ainda o feedback dado a esses especialistas que possibilita a reavaliação de seu posicionamento frente aos resultados obtidos em cada uma das etapas realizadas na técnica Delphi.

Como em diferentes técnicas de pesquisa apresenta as suas desvantagens, destacando a dificuldade de contato com os especialistas e de retorno dos instrumentos, considerando que é previsto para a aplicação dessa técnica, que os sujeitos sejam 
"experts" na área de estudo. Esses especialistas desempenham, na maioria das vezes, diferentes atividades profissionais e de pesquisa, fator que dificulta o retorno dos instrumentos nos prazos determinados, sendo necessário um contato contínuo com os sujeitos.

Esse tipo de técnica, quando aplicada com um rigor de estudo e análise, pode produzir pesquisas com resultados relevantes tanto para teoria quanto para prática profissional em diferentes áreas. Ao passo que profissionais poderão ter disponíveis uma lista de indicadores formulados por especialistas na área, podendo aplicar em suas situações cotidianas rumo à busca pelo desenvolvimento de forma sustentável.

\section{Agradecimentos}

Esta pesquisa teve o apoio financeiro da Fundação de Amparo à Pesquisa do Estado de São Paulo (Fapesp).

\section{REFERÊNCIAS}

ARAÚJO, Geraldo José Ferraresi; CARVALHO, Cesar Machado. A Agenda 21 e a elaboração de Políticas Públicas para Sustentabilidade Urbana. Fórum Ambiental da Alta Paulista, v. 7, n. 4, p. 685-699, 2011.

BELLEN. Hans Michael Van. Indicadores de Sustentabilidade: Uma análise comparativa. Rio de Janeiro: FGV, 2006. 256 p.

CNUMAD. Agenda 21. Curitiba: IPARDES, 2001. 260 p.

COUTINHO, Silvano Silva. Competências do profissional de educação física na Atenção Básica. 2011. 207 f. Tese (Doutorado em Enfermagem em Saúde Pública) Escola de Enfermagem de Ribeirão Preto, Universidade de São Paulo, Ribeirão Preto, 2011.

DESLANDES, Suely Ferreira; MENDES, Corina Helena Figueira; PIRES, Thiago Oliveira; CAMPOS, Daniel Souza. Use of the Nominal Group Technique and the Delphi Method to draw up evalution indicators for strategies to deal with violence against children and 
adolescents in Brazil. Revista Brasileira Saúde Materno Infantil, Recife, v.10, p.529$537,2010$.

GALLOPÍN, Gilberto Carlos. Indicators and Their Use: Information for Decision-making. In: MOLDAN, Bedrich; BILHARZ, Suzanne; MATRAVERS, Robyn. Sustainability Indicators. A Report on the Project on Indicators of Sustainable Development. Scope: Wiley, Chichester, 1997. Cap1, p. 13-27.

GONTIJO, Liliane Parreira Tannús. Construindo as competências do cirurgiãodentista na atenção primária em saúde. 2007. 228 f. Tese (Doutorado em Enfermagem em Saúde Pública) - Escola de Enfermagem de Ribeirão Preto, Universidade de São Paulo, Ribeirão Preto, 2007.

HODGE, Antony R.; HARDI, Peter; BELL, David V. J.. Seeing change though the lens of sustainability. Winnipeg: International Institute for Sustainable Development, 1999.19p.

KAYO, Eduardo Kazuo; SECURATO, José Roberto. Método Delphi: Fundamentos, críticas e vieses. Cadernos de Pesquisa em Administração, São Paulo, v. 1, n. 4, p. 5161, $1^{\circ}$ sem. 1997.

LINSTONE, Harold A.; TUROFF, Murray. The Delphi Method: techniques and applications. California: University of Southern California, 2002. 616 p.

MEADOWS, Donella. Indicators and information systems for sustainable development. South Africa: The Sustainability Institute, 1998. 77 p.

OKOLI, Chitu; PAWLOWSKI, Suzanne D. The Delphi method as a research toll: an example, design consideration and applications. Information \& Management, Amsterdam. 42, p. 15-29, 2004.

PADILHA, Maria Luiza Moraes Leonel. Indicadores de desenvolvimento sustentável para o setor têxtil. 2009. 311 f. Tese (Doutorado em Saúde Pública) - Faculdade de Saúde Pública, Universidade de São Paulo, São Paulo, 2009.

PHILIPPI JR, Arlindo; MALHEIROS, Tadeu Fabrício; AGUIAR, Alexandre Oliveira. O. Indicadores de Desenvolvimento Sustentável. In: PHILIPPI JR, Arlindo. Saneamento, saúde e ambiente: fundamentos para um desenvolvimento sustentável. São Paulo: Manole, cap. 22, p. 761-808, 2005.

POLAZ, Carla Natacha Marcolino; TEIXEIRA, Bernardo Arantes Nascimento. Indicadores de sustentabilidade para a gestão de resíduos sólidos urbanos: um estudo para São Carlos. Engenharia Sanitária e Ambiental, Rio de Janeiro, v. 14, n. 3, p 411-420, jul/set 2009. 
POWELL, Catherine. The Delphi technique: myths and realities. Journal of Advanced Nursing, Oxford, v. 41, n.4, p 376-382, 2002.

RIBEIRO, José Cláudio Junqueira; HELLER, Leo. Indicadores ambientais para países em desenvolvimento. Biblioteca virtual de desarrollo sostenible y salud ambiental. Disponível em: <http://www.bvsde.paho.org/bvsAIDIS/PuertoRico29/junque.pdf>. Acesso em: 2 mar. 2011.

SPÍNOLA, Aracy Witt Pinho. Delfos: proposta tecnológica alternativa. São Paulo: Faculdade de Saúde Pública, USP, 1984. 91 p.

TAKAYANAGUI, Angela Maria Magosso. Trabalhadores de saúde e meio ambiente: ação educativa do enfermeiro na conscientização para gerenciamento de resíduos sólidos. 1993.192 f. Tese (Programa Interunidades de Doutoramento em Enfermagem) Escola de Enfermagem de Ribeirão Preto, Universidade de São Paulo, Ribeirão Preto, 1993.

THOMAS, Jerry R.; NELSON, Jack K.; SILVERMAN, Stephen J. Métodos de pesquisa em atividade física. Porto Alegre: Artmed, 2007. 396 p.

UNITED NATIONS. Department of Economic and Social Affairs. Indicators of sustainable development: guidelines and methodologies.(Third ed.). New York, United Nations, 2007.

VEIGA, José Eli. Desenvolvimento sustentável: O desafio do século XXI. Rio de Janeiro: Garamond, 2010. 220 p.

WITT, Regina Rigatto. Competências da enfermeira na atenção básica: contribuição à construção das funções essenciais de saúde pública. 2005. 336 f. Tese (Doutorado em Enfermagem em Saúde Pública) - Escola de Enfermagem de Ribeirão Preto, Universidade de São Paulo, Ribeirão Preto, 2005.

WRIGHT, James Terence Coulter; GIOVINAZZO, Renata Alves. Delphi: uma ferramenta de apoio ao planejamento prospectivo. Caderno de pesquisas em Administração, São Paulo, v. 01, n. $12,2^{\circ}$ trim. 2000. 